\title{
Gestão estratégica do talento na perspectiva individual e organizacional: Proposta de modelo
}

Strategic talent management in the individual and organizational perspective: Framework proposal Gestión estratégica del talento em una perspectiva individual y organizacional: Proposición de um modelo

\author{
Carla Cristina CARACOL ${ }^{1, a}$ \\ Patrícia Jardim da PALMA ${ }^{a}$ \\ Miguel Pereira LOPES ${ }^{a}$ \\ Maria José SOUSA ${ }^{\mathrm{b}}$ \\ Universidade de Lisboa, Instituto Superior de Ciências Sociais e Políticas, Lisboa, Portugala \\ Universidade Europeia, Lisboa, Portugal ${ }^{\mathrm{b}}$
}

ReSUMO Este ensaio teórico tem como objetivo analisar a gestão estratégica do talento no atual contexto da "nova carreira organizacional”, caracterizado pela responsabilidade partilhada entre o trabalhador e a organização, como resultado das transformações do âmbito competitivo em que operam as empresas e das consequentes mutações no mundo do trabalho. É apresentado um modelo conceitual de análise, baseado em uma revisão de literatura intensiva, que pretende fazer refletir sobre as políticas, processos e práticas de gestão dos recursos humanos promovidas pela organização, ao nível da identificação, desenvolvimento e retenção, materializadas em processos de gestão de talento, em uma perspectiva de alinhamento com atitudes e comportamentos esperados dos trabalhadores. Introduz-se, também, um novo potencial indicador para a identificação de talento interno: os comportamentos moldadores. As proposições apresentadas irão permitir analisar em contexto empírico o sucesso da gestão de talento, em termos de alavancagem de desempenho individual e organizacional, verificando a sua eficácia, possibilitando pistas para processos de melhoria contínua.

Palavras-chave:

Gestão estratégica de talento; moldagem do trabalho; nova carreira organizacional.

\section{Abstract}

This theoretical essay aims to analyze strategic talent management in the context of the "new organizational career", characterized by the shared responsibility between the worker and the organization as a result of the changes in the competitive environment in which organizations act as well as the changes in the working world. A conceptual analysis model is presented, based on intensive literature research that intends to reflect on the policies, processes, and practices of human resources management promoted by the organization to identify, develop, and retain internal talent, aligned with the attitudes and behaviors expected in workers. A new potential indicator to identify internal talent is also introduced: crafter behavior. We present some proposals that will allow us to analyze in empirical context the success of talent management, in terms of individual and organizational performance gains, verifying its effectiveness, offering help in the necessary continuous process improvement.

Keywords:

Talent strategic management; crafter behavior; new organizational career.

Resumen Este ensayo teórico tiene como objetivo analizar la gestión estratégica del talento en el actual contexto de la "nueva carrera organizacional", caracterizado por la responsabilidad compartida entre el empleado y la organización, como resultado de las transformaciones en el ámbito competitivo en el que operan las empresas y los subsequentes câmbios en el mundo del trabajo. Se presenta un modelo conceptual de análisis, basado en una revisión intensiva literatura que pretende reflexionar sobre las políticas, procesos y prácticas de gestión 
de recursos humanos, promovidas éstas por la organización a nivel de la identificación, desarrollo y retención, y materializadas en los procesos de gestión del talento, en alineación con la perspectiva actitudes y comportamientos que se esperan de los trabajadores. Se introduce también, un nuevo indicador potencial para la identificación de talento interno: moldeadores de conducta. Las propuestas presentadas irían permitir analizar en el contexto empírico el éxito de la gestión del talento, en términos del empuje del desempeño individual y organizacional, verificando su eficacia, posibilitando pistas para procesos de mejoria continua.

Palabras-clave:

Gestión estratégica del talento; moldeo del trabajo; nueva carrera organizacional.

$\mathrm{N}$

o contexto de novo paradigma de carreira organizacional, designada "nova carreira organizacional", pautada pela partilha de responsabilidade ao nível da sua gestão entre trabalhador e organização, o grande desafio endereçado à gestão de talento é que as organizações detenham o talento correto no local e tempo certos, com as competências e motivações necessárias, em todos os níveis e em todas as localizações da empresa (Guthridge, Komm, \& Lawson, 2008), tendo os gestores a responsabilidade na sua prossecução, por meio da concepção, desenvolvimento e implementação de políticas, processos e práticas de gestão de recursos humanos, que permitirão atingir os objetivos organizacionais (Collings \& Mellahi, 2009; Lewis \& Heckman, 2006).

É nessa perspectiva que se desenvolve este artigo, com o objetivo de compreender como o talento é gerido estrategicamente nas organizações, em uma forma de arquitetura entre a estratégia e o talento (Gagné, 2010), em que se procura transpor os comportamentos e atitudes dos trabalhadores para um contrato psicológico de natureza relacional, em longo prazo (Collings \& Mellahi, 2009), alinhado com as políticas, processos e práticas em vigor na organização.

Assim, partindo de uma perspectiva de talento segmentado (Becker, Huselid, \& Beatty, 2009), a sua gestão é caraterizada pela identificação sistemática de trabalhadores talentosos, cujo valor diferenciado para a organização conduz a um desenho distinto de planos de desenvolvimento e retenção, em uma abordagem transversal de alinhamento de objetivos individuais com os objetivos organizacionais, constituído, respectivamente, nos próprios desempenhos.

Assume-se, assim, que o talento pode ser adquirido e/ou desenvolvido, tendo a gestão de talento como objetivo a sua alavancagem permanente (Dries \& Pepermans, 2008), promovendo o estabelecimento de uma cultura/clima em que as habilidades são apreciadas, as elevadas expectativas são criadas e o sucesso é antecipado (Meyers, Van Woerkom, \& Dries, 2013). O propósito é fazer sobressair o melhor dos trabalhadores (Meyers et al., 2013), colocando-os em posições onde possam ser desafiados para desenvolver o seu potencial (Buckingham, 2005; Buckingham \& Vosburgh, 2001; Yost \& Chang, 2009).

Por meio da definição de um modelo conceitual de análise, são apresentadas proposições que permitirão verificar as referidas ligações entre conceitos/processos, devidamente desagregados nas quatro fases inerentes à operacionalização da gestão de talento: (a) a gestão estratégica do talento no contexto da nova carreira organizacional, (b) a identificação do talento, (c) o desenvolvimento do talento identificado e (d) a retenção do talento detido.

\section{Gestão estratégica do talento no contexto da "nova carreira organizacional"}

$\mathrm{Na}$ virada do milênio, um novo paradigma de carreira surgiu no contexto organizacional, em virtude da necessidade de os desempenhos individual e institucional estarem alinhados, na medida das necessidades e expectativas de trabalhador e empresa, em uma responsabilização de ambos em oportunidades ditadas e aproveitadas, tendo como objetivo o ganho mútuo.

Esta designada "nova carreira organizacional" combina as características de uma carreira organizacional tradicional com as das boundaryless e das protean careers, pautada pelas seguintes características: (a) a organização não pode oferecer um emprego para toda a vida, mas oferece uma boa proposta de valor, baseada na lealdade mútua; (b) a organização tem de pensar de forma criativa, para ganhar a lealdade dos seus trabalhadores, visando atingir elevados níveis de comprometimento, se quiser reter os seus talentos; (c) os trabalhadores têm que demonstrar capacidade para se adaptar e mudar, sendo mais flexíveis, sabendo lidar com a incerteza; (d) a organização e os trabalhadores terão que aceitar a responsabilidade pelo desenvolvimento e gestão de carreira: por um lado, os trabalhadores deverão definir objetivos e planejar a sua obtenção, aproveitando as 
oportunidades para formação e desenvolvimento existentes na organização, ou externamente; e, por outro lado, a organização deverá apoiar e dar suporte, sempre que possível, a esses objetivos de carreira, por meio de atividades adequadas, como mentoring e mobilidade funcional; (e) o sucesso de carreira passa a ser medido em termos de mobilidade interna, promovendo-se oportunidades para aprendizagem, diversidade de competências e satisfação pessoal; (f) o contrato de trabalho será baseado, também, em uma relação psicológica, promovendo-se o comprometimento e a vontade de investir tempo e esforço em adquirir competências específicas da organização; e (g) considera-se essencial um comportamento protean, que potencialize a aprendizagem e a mudança nos trabalhadores, podendo a organização possibilitar uma carreira que, embora não linear, otimize essas competências adquiridas e desenvolvidas. Pretende-se, acima de tudo, que a relação existente entre organização e trabalhador seja mais positiva, num quadro de ganho mútuo (Clarke, 2013). Consequentemente, este novo paradigma coloca o ônus da gestão de carreira não apenas na organização, mas também, e sobretudo, no trabalhador (Cappelli \& Keller, 2014), o que terá impacto na gestão estratégica dos recursos disponíveis, designadamente os humanos.

Nesse contexto, enquanto novo paradigma de carreira, a "nova carreira organizacional" figura-se como caracterizada por uma responsabilidade partilhada entre o trabalhador e a organização, em que o primeiro aproveita as oportunidades conferidas pela segunda para o seu desenvolvimento; a organização, por sua vez, procura mobilizar o talento do trabalhador, reconhecendo-o.

De modo recorrente, a organização e os trabalhadores terão que aceitar a responsabilidade partilhada pelo desenvolvimento e gestão de carreira, procurando um encontro de expetativas e necessidades de ambos, numa situação de win-win. As organizações atuam assim, para Kong, Cheung e Song (2011), como promotores do desenvolvimento de seus trabalhadores, de forma a aumentar a sua produtividade, o que impactará no aumento das suas competências competitivas no mercado em que atuam (Baruch, 2006).

É nesse contexto que, em pleno século XXI, nos debruçamos sobre a importância contextual no que diz respeito a um tópico cada vez mais popular (Chuai, Preece, \&Iles, 2008), na medida em que a sua propriedade é considerada determinante para o sucesso organizacional (Beechler \&Woodward, 2009; Collings \& Mellahi, 2009; Iles, Preece, \& Chuai, 2010; Meyers et al., 2013; Tarique \& Schuler, 2010), fundamental para a competitividade (Lawler, 2009) e elevado desempenho (Hilthrop, 1999) das organizações: a guerra pelo talento (Michaels, Handfield-Jones, \& Axelrod, 2001).

Michaels et al. (2001) definem talento como um conjunto de habilidades de um trabalhador - seus dons, conhecimentos, experiência, inteligência, discernimento, atitude, caráter, impulsos inatos, incluindo, igualmente, a sua capacidade de aprender e se desenvolver. De forma complementar, Gagné (2000) defende que a detenção de talento permite que quem o possui atue acima dos seus pares, estando, por isso, muitas vezes, associado a desempenhos de excelência (Gallardo-Gallardo, Dries, \& González-Cruz, 2013), pressupondo a existência de três componentes essenciais: competência, compromisso e sentido de contribuição (Ulrich \& Smallwood, 2012).

No entanto, embora seja comumente aceita a importância do talento e da sua gestão adequada, continua a faltar consistência na sua conceitualização, defendendo Lewis e Heckman (2006) que é essencialmente um eufemismo, podendo significar o que o líder do negócio ou escritor quiser (Ulrich, 2007). Dessa forma, por meio do desenvolvimento de um modelo conceitual de análise (Figura 1), é objetivado este conceito, bem como a sua operacionalização, levantando proposições que estabelecem relações entre as três fases da gestão estratégica de talento: sua identificação, desenvolvimento e retenção, num contexto de "nova carreira organizacional" pautado pela expectativa de alinhamento entre as atitudes e os comportamentos do trabalhador com as políticas, processos e práticas da organização.

Parte-se, assim, de uma abordagem baseada na gestão de talento estratégica segmentada (Becker et al., 2009), que é a visão mais comum utilizada nas organizações (Ready, Conger, \& Hill, 2010), tendo como pressuposto base que a alocação de mais recursos para os trabalhadores com potencial e/ou desempenho elevado conduzirá a um maior retorno do investimento (Bothner, Podolny, \& Smith, 2011). 


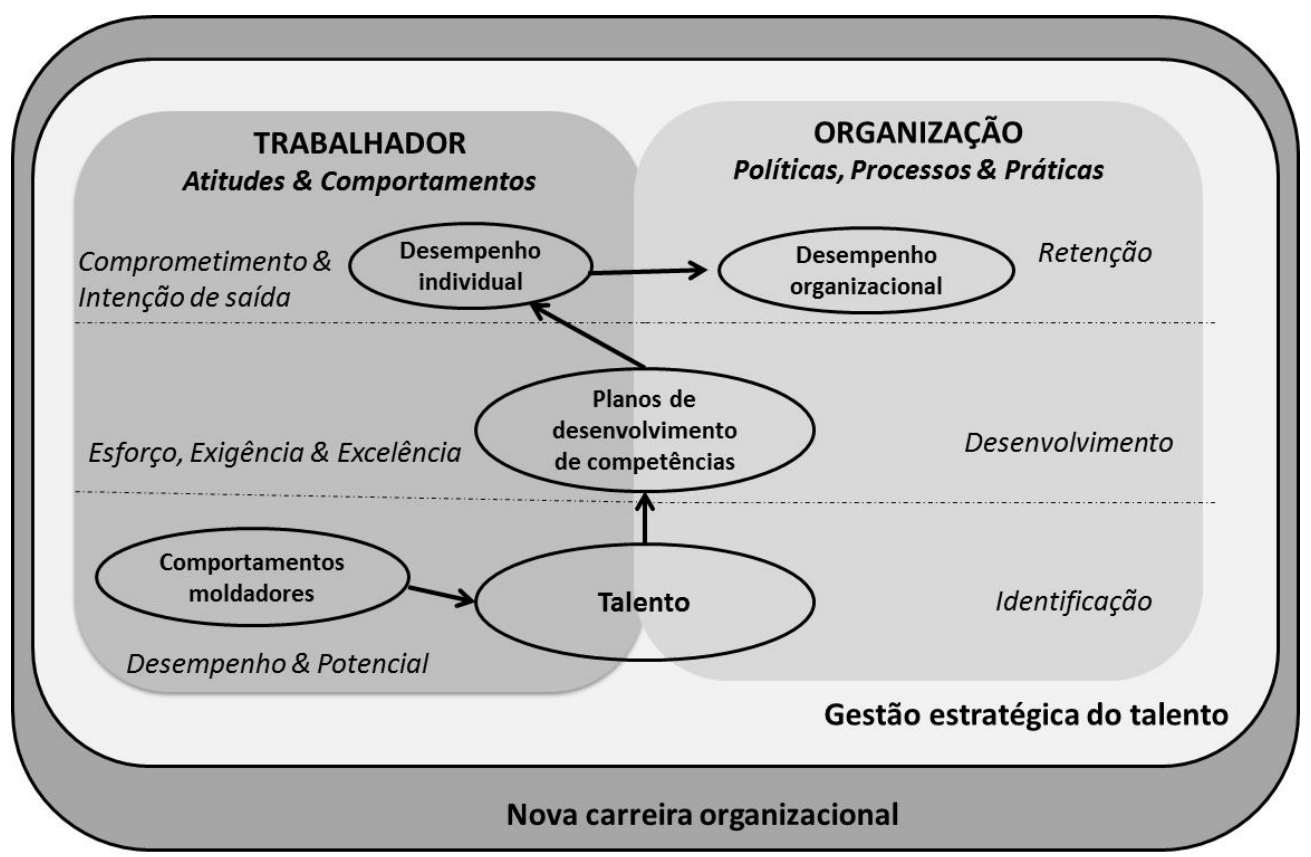

FIGURA 1. Quadro conceitual de análise

\section{A identificação do talento}

$\mathrm{Na}$ atualidade, verifica-se a importância do alinhamento entre o talento individual e o seu contexto de trabalho, em termos de organização e/ou função, encarando-se este em uma perspectiva subjetiva e relativa (Jericó, 2001).

Assim, o talento deve ser operacionalizado de forma situacional, de acordo com a cultura, o ambiente, o tipo de trabalho, mas, sobretudo, com os propósitos de negócio, assumindo uma abordagem segmentada e igualmente, estratégica (Becker et al., 2009).

Nessa linha, denota-se a importância das políticas, processos e práticas de recursos humanos estarem alinhadas com as variáveis organizacionais (Lewis \& Heckman, 2006) no que diz respeito à gestão do talento, enunciando a primeira proposição:

Proposição 1 (P1) - As políticas, processos e práticas de gestão estratégica do talento existentes na organização influenciam a percepção do talento interno detido.

Assim, será a partir de uma correta conceitualização e identificação do talento detido na organização que se conseguirá otimizar o investimento habitual efetuado no capital humano e criar planos de desenvolvimento e retenção adequados para os que se espera contribuírem, de forma positivamente diferenciadora, para o desempenho organizacional.

$\mathrm{E}$, nesse entender, importa verificar quais as manifestações de talento a considerar, salientando Ulrich e Smallwood (2012) que para um trabalhador ser percebido como talentoso, pressupõe-se que tenha competência, compromisso e sentido de contribuição, sendo, por meio desse conjunto, que o seu capital humano se tornará mais produtivo, auxiliando a organização a ser mais capaz frente à mudança, às expectativas dos clientes e mais competitiva em relação aos concorrentes. Será com base nesse pressuposto que se desenvolvem as proposições seguintes, espelhadas na Figura 2.

Por ser a visão mais comum existente e utilizada nas organizações, como também pelos próprios trabalhadores, um estudo realizado por Dries e Pepermans (2008), considerou que os trabalhadores excepcionais pertencem a uma elite, sendo raros. Consequentemente, assume-se que os lotes de talento, ou seja o conjunto de trabalhadores identificados pela organização como talentosos, incluem os trabalhadores de elevado potencial e desempenho (Dries \& Pepermans, 2008; Gallardo-Gallardo et al., 2013), o que, embora sendo duas perspectivas distintas, são complementares. 
$\mathrm{Na}$ segunda proposição apresenta-se o talento percebido como potencial elevado (Silzer \& Church, 2010), definindo-se potencial como as estruturas inobserváveis (Yost \& Chang, 2009) que possibilitam aos trabalhadores se desenvolverem para um nível de proficiência superior face ao atual, defendendo Pepermans, Vloeberghs e Perkisas (2003) que estes demonstram necessidades, motivações e comportamentos diferenciados frente aos trabalhadores medianos:

Proposição 2 (P2) - A organização percebe os trabalhadores com elevado potencial como talentosos.

$\mathrm{Na}$ terceira proposição, o talento é encarado como elevado desempenho (Stahl et al., 2007), correspondendo aos trabalhadores que estão posicionados no topo em termos de capacidade e resultados, demonstrando uma habilidade excepcional de concretização (Williams, 2000):

Proposição 3 (P3) - A organização percebe os trabalhadores com elevado desempenho como talentosos.

Contudo, e apesar da importância das proposições anteriores, os especialistas de recursos humanos devem focar-se não apenas na conceitualização e implementação de decisões relacionadas à identificação do seu talento, mas também à melhoria dessas práticas (Boudreau \& Ramstad, 2005), de forma estrutural e sistemática, acompanhando a hipotética volatilidade organizacional.

Cabe salientar que, concomitantemente, o trabalhador identificado como pertencente ao lote de talento, para além do status organizacional alcançado, tem acesso a um conjunto de ações programadas de desenvolvimento que visam à otimização das suas competências e conhecimentos, permitindo-lhes uma gestão pessoal de carreira mais eficaz e eficiente no sentido de uma maior adaptabilidade a diferentes contextos de trabalho (Sullivan \& Baruch, 2009).

\section{A importância da moldagem do trabalho}

$\mathrm{Na}$ medida em que o contexto pode influenciar os trabalhadores de diferentes formas, Papierno, Ceci, Makel e Williams (2005) defendem que a gestão de talento deve ser dinâmica e adaptável ao contexto e/ou ao trabalhador. A teoria de moldagem do trabalho nos proporciona uma visão complementar à abordagem clássica de identificação de talento, anteriormente apresentada, na medida em que capta as alterações ativas promovidas pelos trabalhadores nos seus descritivos funcionais, no sentido de lhes proporcionar mais-valias, incluindo envolvimento, satisfação no trabalho, resiliência e prosperidade (Berg, Dutton, \& Wrzesniewski, 2007).

Assim, se o trabalhador se limitar a seguir a essência do descritivo funcional de controle burocrático (Strang \& Baron, 1990) que carateriza a função por si desempenhada, com as listas estáticas de tarefas, responsabilidades, relações de reporte, entre outros, concebido em uma perspectiva topo-base (Wrzesniewski, LoBuglio, Dutton, \& Berg, 2013), a sua percepção e interpretação psicológica pode condicionar a demonstração do seu talento pessoal, desperdiçando-o.

Estamos, assim, ao nível da disponibilização de modelos que demonstrem as atividades inerentes à função, em uma relação simplificada de estímulo, resposta esperada e reforço positivo. É o designado modeling, mencionado por Bandura (1971), cujo conceito traduzido se refere a modelagem que, embora seja contextualizada pelo autor na observação de modelos de aprendizagem, será por nós adaptado, no que diz respeito à sua tradução, como o ato de modelar, de delinear, de dar forma, de reproduzir exatamente (Costa \& Sampaio e Melo, 1999). Contudo, o próprio Bandura, em 1971, referia a possibilidade de, complementarmente a essa modelagem, as pessoas, face aos modelos estabelecidos, poderem pautar a sua conduta, igualmente, por comportamentos inovadores, posição reforçada, em contexto organizacional, por Wrzesniewski et al. (2013).

Nessa linha de pensamento, estes autores referem que o descritivo funcional tem impacto expressivo nas experiências psicológicas dos trabalhadores no desempenho da sua função, defendendo Rosso, Dekas e Wrzesniewski (2010) que este deverá ser visto como um ponto de partida, um referencial, podendo o trabalhador aumentar ou diminuir o esforço empregado na procura do seu reforço psicológico (Dutton, Roberts, \& Bednar, 2010), modificando o modelo estabelecido. Estamos, assim, no nível da moldagem, da forma que se refere ao ajustamento, à adaptação frente ao molde/modelo existente (Costa \& Sampaio e Melo, 1999).

Parte-se, portanto, do pressuposto de que o contexto social fornece ao trabalhador os recursos para construir a sua experiência individual de trabalho (Wrzesniewski et al., 2013), considerando-se que parte da 
identificação social é moldada pelo próprio trabalhador (Lyons, 2008), o que impactará na motivação/empenho para o trabalho e no respetivo resultado de desempenho (Wrezesniewski \& Dutton, 2001), podendo-se verificar demonstrações mais frequentes do seu talento pessoal.

Alguns autores defendem que a moldagem do trabalho tem efeitos positivos ao nível do seu bem-estar psicológico (Berg, Grant, \& Johnson, 2010), no seu envolvimento (Tims, Bakker, \& Derks, 2012), sendo importante para os resultados individuais e organizacionais (Wrzesniewski et al., 2013), assumindo-se como equilíbrio instrumental para atingir objetivos de trabalho, aprendizagem e desenvolvimento pessoal $(\mathrm{Hu}$, Schaufeli, \& Taris, 2011).

Bond, Flaxman e Bunce (2008) descrevem o impacto positivo desse redesenho na experiência de trabalho, na medida em que as alterações ativas promovidas pelos trabalhadores no seu descritivo funcional personalizam a sua função para melhor servir seus motivos, forças e paixões (Lyons, 2008).

Consequentemente, se essa moldagem é boa ou ruim para a organização, isso deve ser analisado estratégica e contextualmente (Wrezesniewski \& Dutton, 2001), dependendo do impacto da maneira como os trabalhadores escolhem fazer a moldagem e de que modo esses comportamentos moldadores contribuirão para o desempenho organizacional, isto é, se as suas motivações e identidades estão alinhadas com os objetivos organizacionais (Wrezesniewski \& Dutton, 2001).

Desse modo, levantamos uma nova proposição ao modelo conceitual apresentado, assumindo que o desempenho extrapapel, ou seja, não contemplado no descritivo funcional, pode não ser reconhecido pela organização e/ou pelas chefias/pares (Organ, 1988), muito embora possa ter um efeito positivo no reforço da relação entre o talento e o desempenho organizacional (Collings \& Mellahi, 2009), conduzindo ao desenvolvimento do trabalhador (Wrezesniewski \& Dutton, 2001):

Proposição 4 (P4) - A organização percebe os comportamentos moldadores como manifestações de talento.

Esses moldadores de trabalho personalizam a função, atuando de três formas (Berg et al., 2007, 2010), que se complementam e não são mutuamente excludentes: (a) alteram as fronteiras do seu trabalho, assumindo uma ou mais tarefas ou alterando sua forma de execução - são as designadas moldagens de tarefa (Wrzesniewski et al., 2013); (b) mudam as suas relações no trabalho, alterando a natureza ou a extensão dessas interações e a dinâmica interpessoal existente, de forma a introduzir significado às suas vidas (Baumeister \& Leary, 1995) são as designadas moldagens de relações (Wrzesniewski et al., 2013); (c) alteram cognitivamente a sua função, modificando a forma como percebem as tarefas, estando motivados para criar uma autoimagem positiva no seu trabalho, procurando atribuir-lhe um significado positivo (Rosso et al., 2010) e construir uma identidade positiva com a organização (Dutton et al., 2010) - são as designadas moldagens cognitivas (Wrzesniewski et al., 2013). Nessa linha, poderemos verificar se a organização percebe de forma individualizada, ou não, essas manifestações moldadoras de trabalho como talento, enunciando-se, respetivamente, três subproposições:

Proposição 4.1 (P4.1) - A organização percebe a moldagem da tarefa como manifestação de talento; Proposição 4.2 (P4.2) - A organização percebe a moldagem da relação como manifestação de talento; Proposição 4.3 (P4.3) - A organização percebe a moldagem cognitiva como manifestação de talento.

Se realizada da melhor forma, a moldagem do trabalho é um meio de os trabalhadores melhorarem suas vidas no exercício da função, com a utilização do talento pessoal, passando o seu envolvimento por um processo participativo que aumenta o comprometimento com o sucesso da organização (Robbins \& Judge, 2013).

Será da competência dos gestores o desafio de fomentar a moldagem de trabalho positiva e evitar a negativa (Berg et al., 2007), devendo ser uma preocupação real e permanente, na medida em que existem evidências consideráveis que ambas as formas de moldagem de trabalho acontecem (Lyons, 2008; Wrezesniewski \& Dutton, 2001), ocorrendo de forma transversal, desde as funções mais simples às mais complexas (Berg et al., 2010), devendo ser, por isso, gerenciável. 


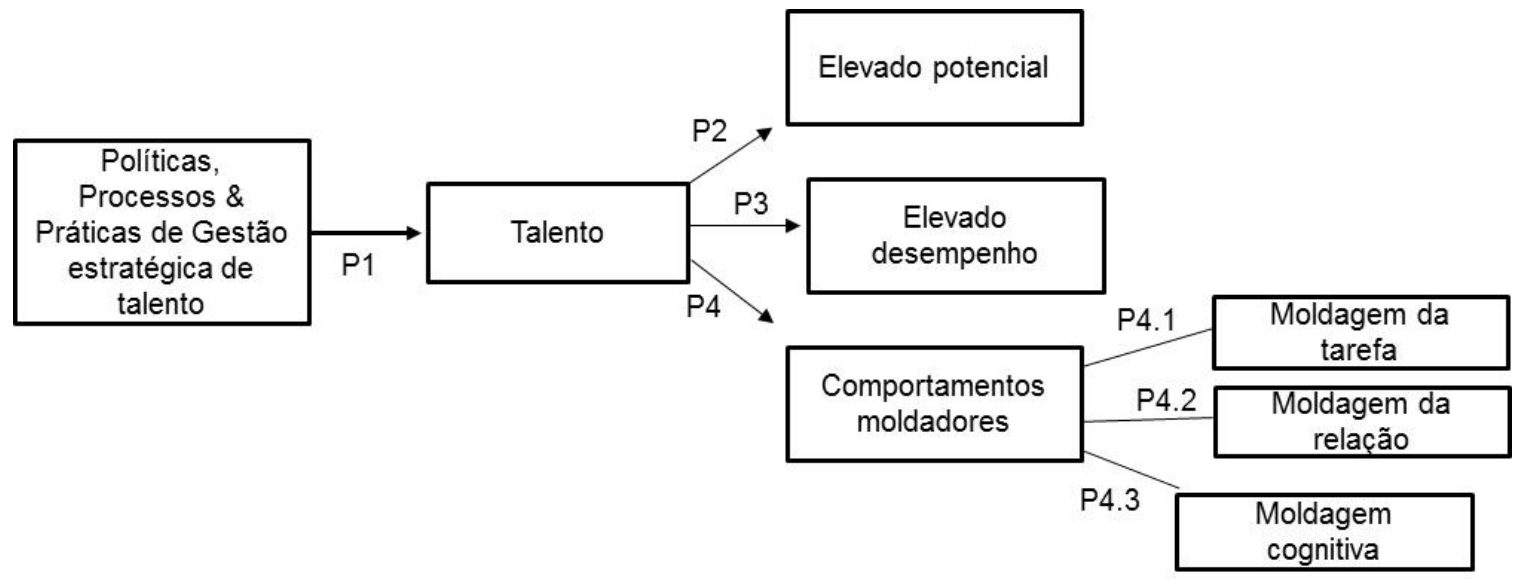

FIGURA 2. Sistematização das proposições relativas à identificação de talento

\section{O desenvolvimento do talento: A mutualização da responsabilidade e do benefício entre trabalhador e organização}

Gagné (2010) defende que a motivação é essencial para o talento, porque o seu processo de desenvolvimento requer exigência sistemática e contínua, com esforço, passando à sua otimização na procura pela excelência, por ter motivos para desencadear a ação que permita que esse talento se manifeste, assegurando que os indivíduos trabalhem até o topo do seu potencial (Redford, 2005).

Parte-se, portanto, da premissa de que o talento pode ser adquirido e/ou potencializado, tendo como objetivo incrementá-lo sistematicamente, de forma a geri-lo melhor, com claros benefícios para a organização, de modo que esse talento seja orientado ao nível da sua carreira, por meio de estratégias e práticas de atuação, como formação, gestão de competências, lançamento de desafios e/ou experiências precoces de liderança, rotação de funções, coaching, mentoring (Dries \& Pepermans, 2008) ou aprendizagem no trabalho (McCall, 2010).

Sobretudo, para o trabalhador detentor de talento, desenvolvimento significa orientação, feedback e aconselhamento. O propósito é evidenciar o melhor dos trabalhadores (Meyers et al., 2013), colocando-os em posições onde possam ser desafiados a desenvolver o seu potencial (Buckingham, 2005; Yost \& Chang, 2009), devendo a gestão de talento promover o estabelecimento de uma cultura/clima que o assegure.

Consequentemente surge uma quinta proposição refletida no modelo conceitual proposto, a qual se baseia na teoria de que em uma perspectiva estratégica, a gestão do talento deverá focalizar-se na melhoria da eficiência e eficácia da organização (Boselie, Dietz, \& Boon, 2005), por meio do desenvolvimento pessoal do seu lote de talento:

Proposição 5 (P5) - Os objetivos estratégicos prosseguidos com a gestão do talento influenciam a conceitualização, desenvolvimento e implementação dos planos de desenvolvimento de competências a operacionalizar na organização.

Nessa perspectiva, os sistemas de gestão de recursos humanos devem ser eficazes no desenvolvimento do seu lote de talento, o qual, de forma inversamente proporcional a sua dimensão restrita, contribui para os resultados organizacionais (Aguinis, Gottfredson, \& Joo, 2012), sendo esperada uma alavancagem do seu desempenho (Figura 3).

E, nesse novo paradigma de carreira organizacional abordado, acreditamos ser importante referir as teorias de Chudzikowski (2012) e Dries (2013) pautadas pela ideia de que a carreira continua a ser importante: para os trabalhadores, o sucesso na carreira pode conduzi-los ao bem-estar (Burke, Burgess, \& Fallon, 2006; Leung, Cheung, \& Liu, 2011) por meio de um melhor desempenho individual; para as organizações, o sucesso das carreiras dos indivíduos contribuirá para a proficiência do desempenho organizacional ( $\mathrm{Ng}$, Eby, Sorenson, \& Feldman, 2005).

Surge, assim, a sexta proposição do modelo conceitual, sugerindo que as oportunidades internas facilitam a troca de informação necessária entre organização e o trabalhador, de forma a relacionar necessidades e expectativas em uma plataforma comum de mercado para talento interno (Cappelli \& Keller, 2014): 
Proposição 6 (P6) - Os planos de desenvolvimento de competências disponibilizados pela organização aos trabalhadores talentosos pressupõem o seu aproveitamento por parte destes, impactando positivamente em seu desempenho individual.

\begin{tabular}{|c|c|c|c|}
\hline $\begin{array}{c}\text { Políticas, Processos \& } \\
\text { Práticas de Gestão } \\
\text { estratégica de talento }\end{array}$ & P5 & $\begin{array}{c}\text { Planos de } \\
\text { desenvolvimento de } \\
\text { competências }\end{array}$ & P6 (+) \\
\cline { 3 - 4 } & & $\begin{array}{c}\text { Desempenho } \\
\text { individual }\end{array}$ \\
\hline
\end{tabular}

FIGURA 3. Sistematização das proposições relativas ao desenvolvimento de talento

Ao serem integrados em programas de desenvolvimento de talento, os trabalhadores aperfeiçoam conhecimentos e competências, flexibilizando seu perfil profissional, tornando-se mais capazes, por meio do estímulo à sua autoestima e confiança, permitindo enfrentar com sucesso os desafios no mercado, reforçando o seu status na organização e, não raras vezes, na própria sociedade.

\section{A retenção do talento: Alinhamento de expectativas e necessidades entre trabalhador e organização}

Assistimos implicitamente, na gestão estratégica de talento, à ideia de exclusividade (Iles et al., 2010; Ready et al., 2010), partindo-se do pressuposto de que a alocação de mais recursos para trabalhadores talentosos na organização conduz a maior retorno do investimento (Bothner et al., 2011), por meio de um aproveitamento mais proficiente das suas capacidades e habilidades, através da gestão interna das suas competências.

É que, muito embora as organizações possam não conseguir efetuar com a frequência desejável o planejamento individual de carreira, o desenvolvimento de competências permanece essencial, na medida em que providencia as estruturas inerentes às oportunidades na qual a mobilidade pode ocorrer (Cappelli \& Keller, 2014), e se crê poderem influenciar, de forma positiva, o desempenho organizacional. E é nesse contexto que propomos uma nova proposição:

Proposição 7 (P7) - Os trabalhadores pertencentes ao lote de talento sujeitos aos planos de desenvolvimento de competências, por meio do seu desempenho individual, alavancam o desempenho organizacional.

Contudo, concomitantemente, importa que o impacto desse investimento positivamente diferenciador seja contínuo, enfatizando-se a retenção desse talento crítico, por meio do estímulo ao comprometimento e à diminuição da sua intenção de saída (Figura 4), promovendo-se a alteração de um contrato psicológico de natureza transacional, a curto termo, para um relacional, a longo termo (Collings \& Mellahi, 2009).

Collings e Mellahi (2009) defendem que o comprometimento é uma ponte essencial entre a gestão de talento e o desempenho organizacional, sendo este um estado psicológico caracterizador da relação estabelecida entre trabalhador e organização (Allen \& Meyer, 2000), que, quando elevado, faz com que os trabalhadores estejam dispostos a dar mais de si próprios, de forma a contribuir para o bem-estar organizacional (Mowday, Porter, \& Steers, 1982). Sugere-se, assim, uma nova proposição:

Proposição 8 (P8) - O desempenho individual do lote de talento interno, desenvolvido, impactará positivamente no desempenho organizacional apenas se o trabalhador estiver comprometido com a organização.

Por outro lado, verificando-se um elevado comprometimento organizacional, alinhando as expectativas e as necessidades de organização e trabalhador, acredita-se que a retenção do trabalhador talentoso possa ser alavancada, na medida em que ele, sujeito a planos de desenvolvimento de competências, detém uma percepção de sucesso futuro (Huselid, 1995; Paré, Tremblay, \& Lalonde, 2001). Nessa medida, sugerimos uma última proposição ao modelo conceitual apresentado:

Proposição 9 (P9) - Os trabalhadores talentosos, que detêm elevado comprometimento organizacional, apresentam menor intenção de saída voluntária. 


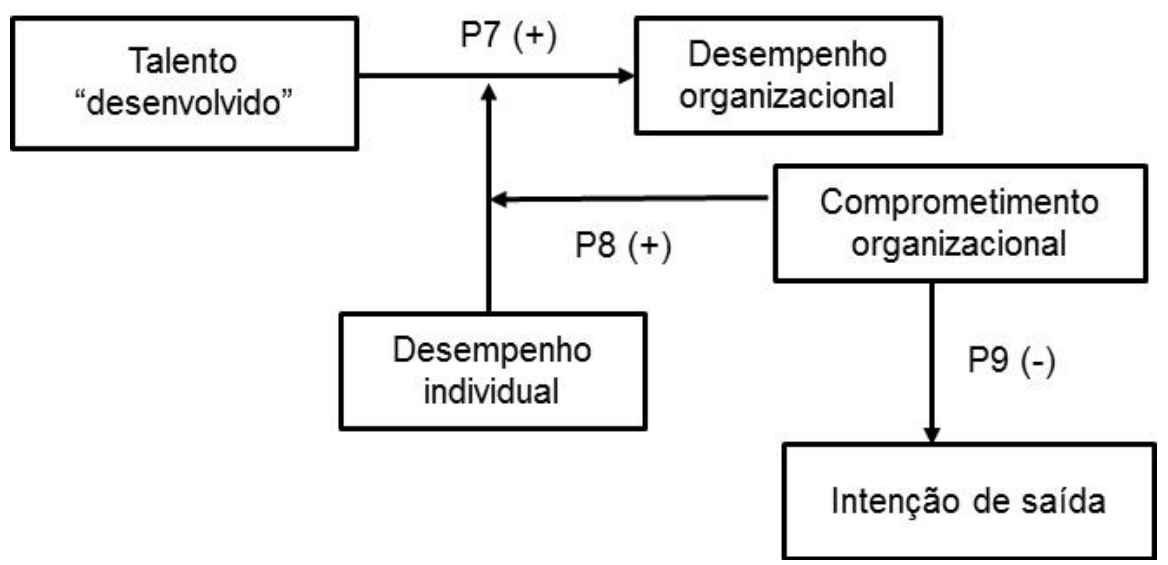

FIGURA 4. Sistematização das proposições relativas à retenção de talento

Questão central na retenção do talento organizacional é o respectivo reconhecimento e recompensa (Cappelli, 1999), devendo o talentoso sentir-se naturalmente comprometido e com sentido de lealdade para com a organização que o apoiou no seu desenvolvimento, lançando-lhe desafios que o estimulassem e providenciando oportunidades que confirmassem o seu potencial.

Já existe pesquisa sobre as questões relacionadas à gestão de talento, porém, com pouco foco na sua aplicabilidade na gestão de recursos humanos, encarando-se o construto talento como um conceito genérico, baseado, sobretudo, em experiência de consultorias (Tarique \& Schuler, 2010). Nessa medida, este artigo teve como propósito analisar, em uma perspectiva pragmática de operacionalização, as etapas inerentes à gestão estratégica do talento - identificação, desenvolvimento e retenção - espelhadas na literatura disponível, apresentando um modelo conceitual de análise que permita verificar, empiricamente, as relações estabelecidas entre variáveis introduzidas. Será esse o objetivo de investigação futura: operacionalizar o citado modelo nas organizações que já dispõem de políticas, processos e práticas em uma perspectiva estratégica de gestão de talento (GallardoGallardo et al., 2013; Gelens, Dries, Hofmans, \& Pepermans, 2013).

Propõe-se, assim, a realização de estudos empíricos longitudinais, que tentem encontrar variáveis e indicadores comuns para uma maior fidedignidade e confiabilidade do conceito de talento, apostando-se assim em um modelo que descreva a gestão de talento com antecedentes e consequentes, atendendo às inerentes estruturas organizacionais de suporte (Thunnissen, Boselie, \& Fruytier, 2013), na emergência da "nova carreira organizacional”. O propósito é, atendendo a uma perspectiva sinérgica de otimização de esforços e recursos disponíveis em ambientes de maior volatilidade e mutação, analisar as caraterísticas dos contratos psicológicos estabelecidos num contexto de lealdades múltiplas.

Concomitantemente, salientamos a importância do aprofundamento do estudo dos comportamentos moldadores enquanto possíveis indicadores de manifestação de talento percebido pela organização. Além disso, verificar de que forma a instituição deve orientar e conduzir o trabalhador, mesmo que indiretamente, aproveitando este ato voluntarioso, que não implica investimento inicial, mas que deverá ser analisado em uma perspectiva do que se poderá retirar como mais-valia organizacional em termos da atitude e do comportamento pretendidos.

Conceitualizar talento, definir métricas para a sua identificação, analisar as políticas, processos e práticas inerentes ao seu desenvolvimento e retenção, na perspectiva do impacto percebido ao nível do desempenho individual por parte do lote de talentosos e suas chefias diretas e definir métricas para a avaliação do seu retorno do investimento efetivo ao nível do desempenho organizacional são alguns dos desafios para investigações futuras, que permitirão enriquecer o conhecimento teórico nesse domínio e, de modo complementar, potencializar a sua aplicabilidade em contextos de trabalho.

\section{REFERÊNCIAS}

Aguinis, H., Gottfredson, R. K., \& Joo, H. (2012). Using performance management to win the talent war. Business Horizons, 55(6), 609-616. doi:10.1016/j.bushor.2012.05.007 
Allen, N. J., \& Meyer, J. P. (2000). Construct validation in organizational behavior research: The case of organizational commitment. In R. D. Goffin \& E. Helmes (Eds.), Problems and solutions in human assessment (pp. 285-314). New York: Springer. doi: 10.1007/978-1-4615-4397-8_13

Bandura, A. (1971). Social learning theory. New York: General Learning Press.

Baruch, Y. (2006). Career development in organizations and beyond: Balancing traditional and contemporary viewpoints. Human Resource Management Review, 16(2), 125-138. doi:10.1016/j.hrmr.2006.03.002

Baumeister, R., \& Leary, M. (1995). The need to belong: Desire for interpersonal attachments as a fundamental human motivation. Psychological Bulletin, 117(3), 497-529. doi:10.1037/0033-2909.117.3.497

Becker, B., Huselid, M., \& Beatty, R. (2009). The differentiated workforce: Transforming talent into strategic impact. Boston: Harvard Business Press.

Beechler, S., \& Woodward, I. C. (2009). The global "war for talent". Journal of International Management, 15(3), 273-285. doi: 10.1016/j.intman.2009.01.002

Berg, J. M., Dutton, J. E., \& Wrzesniewski, A. (2007). What is job crafting and why does it matter? Michigan Ross School of Business, 1-8.

Berg, J., Grant, A., \& Johnson, V. (2010). When callings are calling: Crafting work and leisure in pursuit of unanswered occupational callings. Organization Science, 21(5), 973-994. doi: 10.1287/orsc.1090.0497

Bond, F. W., Flaxman, P. E., \& Bunce, D. (2008). The influence of psychological flexibility on work redesign: Mediated moderation of a work reorganization intervention. Journal of Applied Psychology, 93(3), 645-54. doi: 10.1037/0021-9010.93.3.645

Boselie, P., Dietz, G., \& Boon, C. (2005). Commonalities and contradictions in HRM and performance research. Human Resource Management Journal, 15(3), 67-94. doi: 10.1111/j.1748-8583.2005.tb00154.x

Bothner, M., Podolny, J., \& Smith, E. (2011). Organizing contest for status: The Mattew effect vs. the Mark effect. Management Science, 57(3), 439-457. doi: 10.1287/mnsc.1100.1281

Boudreau, J. W., \& Ramstad, P. M. (2005). Talentship, talent segmentation, and sustainability: A new HR decision science paradigm for a new strategy definition. Human Resource Management, 44(2), 129-136. doi: 10.1002/hrm.20054

Buckingham, M. (2005). What great managers do. Harvard Business Review, 83, 70-79.

Buckingham, M., \& Vosburgh, R. M. (2001). The 21st century human resources function: It's the talent, stupid! People and Strategy, 24(4), 17.

Burke, R., Burgess, Z., \& Fallon, B. (2006). Organizational practices supporting women and their satisfaction and well-being. Women in Management Review, 21(5), 416-425. doi: 10.1108/09649420610676217

Cappelli, P. (1999). Career jobs are dead. California Management Review, 42(1), 146. doi: 10.2307/41166023

Cappelli, P., \& Keller, J. R. (2014). Talent management: Conceptual approaches and practical challenges. Annual Review of Organizational Psychology and Organizational Behavior, 1(1), 305-331. doi: 10.1146/annurev-orgpsych-031413-091314

Chuai, X., Preece, D., \& lles, P. (2008). Is talent management just old wine in new bottles? Management Research News, 31(12), 901-911. doi: 10.1108/01409170810920611

Chudzikowski, K. (2012). Career transitions and career success in the "new" carrer era. Journal of Vocational Behavior, 81(2), 298-306. doi: 10.1016/j.jvb.2011.10.005

Clarke, M. (2013). The organizational career: Nor dead but in need of redefinition. The International Journal of Human Resource Management, 24(4), 684-703. doi:10.1080/09585192.2012.697475

Collings, D. G., \& Mellahi, K. (2009). Strategic talent management: A review and research agenda. Human Resources Management Review, 19(4), 304-313. doi:10.1016/j.hrmr.2009.04.001

Costa, J. A., \& Sampaio e Melo, A. (1999). Dicionário da Língua Portuguesa. Porto: Porto Editora.

Dries, N. (2013). The psychology of talent management: A review and research agenda. Human Resource Management Review, 23, 272-285. doi: 10.1016/j.hrmr.2013.05.001

Dries, N., \& Pepermans, R. (2008). "Real" high-potential-careers: An empirical study into the perspectives of organisations and high potentials. Personnel Review, 37(1), 85-108. doi: 10.1108/00483480810839987

Dutton, J., Roberts, L., \& Bednar, J. (2010). Pathways to positive identity construction at work: Four types of positive inetity and the building of social resources. Academy of Management Review, 35(2), 265-293.

Gagné, F. (2000). Understanding the complex choreography of talent development through DMGT-Based analysis. Oxford: Elsevier. 
Gagné, F. (2010). Motivation within the DGMT 2.0 framework. High Ability Studies, 21(2), 81-99. doi: $10.1080 / 13598139.2010 .525341$

Gallardo-Gallardo, E., Dries, N., \& González-Cruz, T. F. (2013). What is the meaning of 'talent'in the world of work? Human Resource Management Review, 23(4), 290-300. doi: 10.1016/j.hrmr.2013.05.002

Gelens, J., Dries, N., Hofmans, J., \& Pepermans, R. (2013). The role of perceived organizational justice in shaping the outcomes of talent management: A research agenda. Human Resource Management, 23(2013), 341-353. doi: 10.1016/j. hrmr.2013.05.005

Guthridge, M., Komm, A., \& Lawson, E. (2008). Making talent management a strategic priority. The McKinsey Quarterly, 1, 49-59.

Hilthrop, J. (1999). The quest for the best: Human resource practices to attract and retain talent. European Management Journal, 17(4), 422-430. doi: 10.1016/S0263-2373(99)00022-5

Hu, Q., Schaufeli, W. B., \& Taris, T. W. (2011). The job demands-resources model: An analysis of additive and joint effects of demands and resources. Journal of Vocational Behavior, 79(1), 181-190.

Huselid, M. A. (1995). The impact of human resource management practices on turnover, productivity, and corporate financial performance. Academy of management journal, 38(3), 635-672. doi: 10.2307/256741

Iles, P., Preece, D., \& Chuai, X. (2010). Talent management as a management fashion in HRD: Towards a research agenda. Human Resource Development International, 13(2), 125-145. doi: 10.1080/13678861003703666

Jericó, P. (2001). La gestión del talento: Enfoque conceptual y empírico. Boletín de Estudios Económicos, 174, 423-441.

Kong, H., Cheung, C., \& Song, H. (2011). Hotel career management in China: Developing a measurement scale. International Journal of Hospitality Management, 30(1), 112-118. doi: 10.1016/j.ijhm.2010.07.003

Lawler, E. (2009). Make human capital a source of competitive advantage. Organizational Dynamics, 38(1), 1-7. doi: 10.1016/j. orgdyn.2008.10.007

Leung, A., Cheung, Y., \& Liu, X. (2011). The relations between life domain satisfaction and subjective well-being. Journal of Managerial Psychology, 26(2), 155-169. doi: 10.1108/02683941111102182

Lewis, R. E., \& Heckman, R. J. (2006). Talent management: A critical review. Human Resource Management Review, 16(2), 139154. doi: 10.1016/j.hrmr.2006.03.001

Lyons, P. (2008). The crafting of Jobs and individual differences. Journal of Business and Psychology, 23(1-2), 25-36. doi: $10.1007 / \mathrm{s} 10869-008-9080-2$

McCall, M. (2010). Recasting leadership development. Industrial and Organizational Psychology, 3(1), 3-19. doi: 10.1111/j.17549434.2009.01189

Meyers, M. C., Van Woerkom, M., \& Dries, N. (2013). Talent - Innate or acquired? Theoretical considerations and their implications for talent management. Human Resource Management Review, 23(2013), 305-321. doi: 10.1016/j. hrmr.2013.05.003

Michaels, E., Handfield-Jones, H., \& Axelrod, B. (2001). The war for talent. Boston: Harvard Business School Press.

Mowday, R., Porter, L., \& Steers, R. (1982). Employee-organizations linkages: The psychology of commitment, absenteeism, and turnover. New York: Academic Press.

Ng, T., Eby, L., Sorenson, K., \& Feldman, D. (2005). Predictors of objective and subjective carrer sucess: A meta-analysis. Personnel Psychology, 58(2), 367-408. doi: 10.1111/j.1744-6570.2005.00515

Organ, D. W. (1988). Organizational citizenship behavior: The good soldier syndrome. Lanham: Lexington Books.

Papierno, P., Ceci, S., Makel, M., \& Williams, W. (2005). The nature and nurture of talent: A bioecological perspective on the ontogeny of exceptional abilities. Journal for the Education of the Gifted, 28(3-4), 312-332. doi: 10.4219/jeg-2005-343

Paré, G., Tremblay, M., \& Lalonde, P. (2001). Workforce retention: What do IT employees really want? In M. Serva (Ed.), Proceedings of the 2001 ACM SIGCPR conference on computer personnel research (pp. 1-10). New York: Association for Computing Machinery. doi: 10.1145/371209.371208

Pepermans, R., Vloeberghs, D., \& Perkisas, B. (2003). High potential identification polices: An empirical study among Belgian companhies. The Journal of Management Development, 22(8), 660-678. doi: 10.1108/02621710310487846

Ready, D., Conger, J., \& Hill, L. (2010). Are you high-potential? Harvard Business Review, 88(6), 78-84.

Redford, K. (2005). Shedding light on talent tactics. Recuperado de http://www.personneltoday.com/hr/shedding-light-on-talenttactics/

Robbins, S. P., \& Judge, T. A. (2013). Organizational behavior (15a ed.). Indianapolis: Pearson Education. 
Rosso, B., Dekas, K., \& Wrzesniewski, A. (2010). On the meaning of work: A theoretical integration and review. Research in Organizational Behavior, 30(2010), 91-127. doi: 10.1016/j.riob.2010.09.001

Silzer, R., \& Church, A. (2010). Identifying and assessing high-potential talent: Current organizational practices. In R. Silzer \& B. Dowell (Eds.), Strategy-driven talent management: A leadership imperative (pp. 213-279). San Francisco: JosseyBass.

Stahl, G., Bjorkman, I., Farndale, E., Morris, S., Paauwe, J., \& Stiles, P., ... Wright, P. M. (2007). Global talent management: How leading multinationals build and sustain their talent pipeline. Fontainebleau: INSEAD The Business School for the World. Recuperado de http://www.insead.edu/facultyresearch/research/doc.cfm?did=2738

Strang, D., \& Baron, J. (1990). Categorical imperatives: The structure of job titles in California state agencies. American Sociological Review, 55(4), 479-495. doi: 10.2307/2095802

Sullivan, S. E., \& Baruch, Y. (2009). Advances in career theory and research: A critical review and agenda for future exploration. Journal of management, 35(6), 1542-1571. doi: 10.1177/0149206309350082

Tarique, I., \& Schuler, R. S. (2010). Global talent management: Literature review, integrative framework, and suggestions for further research. Journal of World Business, 45(2), 122-133. doi: 10.1016/j.jwb.2009.09.019

Thunnissen, M., Boselie, P., \& Fruytier, B. (2013). Talent management and the relevance of context: Towards a pulralistic approach. Human Resource Management Review, 23(4), 326-336. doi: 10.1016/j.hrmr.2013.05.004

Tims, M., Bakker, A. B., \& Derks, D. (2012). Development and validation of the job crafting scale. Journal of Vocational Behavior, 80(1), 173-186. doi: 10.1016/j.jvb.2011.05.009

Ulrich, D. (2007). The talent trifecta. Recuperado de http://www.workforce.com/articles/the-talent-trifecta

Ulrich, D., \& Smallwood, N. (2012). What is talent? Leader to Leader, 2012(63), 55-61.

Williams, M. (2000). The war for talent: Getting the best from the best. Londres: Chartered Institute of Personnel \& Development.

Wrezesniewski, A., \& Dutton, J. E. (2001). Crafting a job: Revisioning employees as active crafters of their work. Academy of Management Review, 26(2), 179-201. doi: 10.5465/AMR.2001.4378011

Wrzesniewski, A., LoBuglio, N., Dutton, J. E., \& Berg, J. M. (2013). Job Crafting and cultivating positive meaning and identity in work. Advances in Positive Organizational Psychology, 1, 281-302. doi: 10.1108/S2046-410X(2013)0000001015

Yost, P. R., \& Chang, G. (2009). Everyone is equal, but some are more equal than others. Industrial and Organizational Psychology, 2(4), 442-445. doi: 10.1111/j.1754-9434.2009.01171 